\title{
Probing the plasmid paradox
}

\author{
This month we celebrate plasmids as drivers of microbial ecology and evolution, and highlight active areas of \\ research.
}

$P$ lasmids are self-replicating genetic elements that coexist with chromosomes within cells, typically in bacteria, but also in archaea and even in some eukaryotes. Their biology and diversity open up a multitude of interesting evolutionary and ecological questions.

In addition to genes necessary for their own replication, plasmids carry DNA that may allow their bacterial hosts to survive challenges or expand their ecological niche. For example, plasmids often carry genes that encode antibiotic resistance. Sharing of these genes among bacteria via horizontal gene transfer leads to the rapid spread of bacteria able to survive antibiotic treatment. Similarly, plasmids often carry virulence genes that facilitate the transition to pathogenicity, or factors that promote the evolution of endosymbiosis when transferred to a new host, therefore enabling the evolution of new bacterial lifestyles.

While plasmid-mediated transmission of genes may be advantageous to the host under certain conditions, essential functions associated with plasmid maintenance, such as replication, conjugation and gene expression, incur a cost to the host. Indeed, models predict the long-term loss of plasmids as well as integration of beneficial genes into the host genome. Thus, it is hard to explain the widespread prevalence of plasmids in bacterial genomes, a phenomenon known as the plasmid paradox. Solving the plasmid paradox is an active area of research, including understanding the mechanisms that underlie plasmid fitness costs as well as compensatory evolution that reduces the burden of plasmid carriage.

In a paper published in Nature Ecology \& Evolution Loftie-Eaton et al. ${ }^{1}$ used experimental evolution of Pseudomonas sp. nov. $\mathrm{H} 2$ carrying multidrug resistance plasmid RP4 to show that the bacterial host evolved towards increased plasmid permissiveness, which involved changes in helicase-plasmid interactions. Also in this journal, Jordt et al. ${ }^{2}$ showed how host-plasmid coevolution of two plasmids conferring environmental drug resistance in communities of Escherichia coli and Klebsiella pneumoniae under antibiotic selection promoted plasmid persistence and the evolution of multidrug resistance bacteria. More recently, Hall et al. uncovered the molecular mechanisms underlying the fitness costs of two plasmids of Pseudomonas fluorescens. The authors link fitness costs of carrying plasmids to their interference with SOS response pathways that are central to the cell's physiology and pinpoint compensatory mutations in single genes located either in the plasmid or the chromosome that ameliorate the cost of the plasmid disruption.

One defining feature of plasmid biology is that most cells carry several copies of the same plasmid. The multicopy nature of plasmids is reflected, among other things, in increased genetic variability and gene expression, with implications for bacterial evolution (reviewed by Rodríguez-Beltrán et al. ${ }^{4}$ ). For example, multicopy plasmids can potentiate evolution of antibiotic resistance by copy number variation - not only can multicopy plasmids increase the rate of resistant mutations, they can also amplify their effect via increased gene expression, as exemplified in $E$. coli carrying the $b l a_{\text {TEM-1 }}$ $\beta$-lactamase gene in a study by San Millan et al. ${ }^{5}$. A study by Rodríguez-Beltrán et al. using the same system, also published in our journal, showed how multicopy plasmids can play a role in evolutionary innovation ${ }^{6}$. In this case, heterozygosity brought about by multicopy plasmids solved trade-offs between ancestral and innovative gene functions, facilitating the evolution of resistance to a new antibiotic.
In addition to antibiotic resistance, plasmids can carry genes that code for extracellular proteins, many of which benefit not only the carrying cell but also others nearby. Such genes can be considered public goods in a group of cells that cooperate to produce them. However, cooperation is challenged by cheaters - cells that benefit from public goods but avoid the cost of producing them - which can eventually outcompete cooperative cells. Because plasmids can transfer genes among cells, including those genes coding for public goods, plasmid-carriage of cooperative genes could potentially stabilize bacterial cooperation. In this issue, Dewar et al. present evidence that goes against this hypothesis. Using phylogenetically controlled comparative analysis, the authors show that genes that encode public goods are not more likely in plasmids than in bacterial chromosomes, nor are they more prevalent in plasmids that transfer at higher rates. Instead, the authors find that plasmids, especially those on broad host-range pathogens, carry many pathogenicity genes, suggesting that plasmid-mediated mobility allows movement of genes that facilitate pathogenicity.

Plasmid research is thriving, and at Nature Ecology \& Evolution we look forward to further understanding how plasmids shape the diversity and ecological success of their hosts, including not only bacteria but also archaea and eukaryotes.

Published online: 2 December 2021 https://doi.org/10.1038/s41559-021-01613-x

\footnotetext{
References

1. Loftie-Eaton, W. et al. Nat. Ecol. Evol. 1, 1354-1363 (2017).

2. Jordt, H. et al. Nat. Ecol. Evol. 4, 863-869 (2020).

3. Hall, J. P. J. et al. PLoS Biol. 19, e3001225 (2021).

4. Rodríguez-Beltrán, J. et al. Nat. Rev. Microbiol. 19, 347-359 (2021).

5. San Millan, A. et al. Nat. Ecol. Evol. 1, 0010 (2017).

6. Rodríguez-Beltrán, J. et al. Nat. Ecol. Evol. 2, 873-881 (2018).

7. Dewar, A. E. et al. Nat. Ecol. Evol. https://doi.org/g5nz (2021).
} 